Pacific Journal of Mathematics

ON THE NUMBER OF POLYNOMIALS OF AN IDEMPOTENT 


\title{
ON THE NUMBER OF POLYNOMIALS OF AN IDEMPOTENT ALGEBRA, II
}

\author{
G. GRÄTZER AND J. PlonkA
}

In part I of this paper a conjecture was formulated according to which, with a few obvious exceptions, the sequence $\left\langle p_{n}(\mathfrak{U})\right\rangle$ of an idempotent algebra is eventually strictly increasing. In this paper this conjecture is verified for idempotent algebras satisfying $p_{2}(\mathfrak{U})=0, p_{3}(\mathfrak{U})>0$, and $p_{4}(\mathfrak{U})>0$. In fact, somewhat more is proved:

Theorem. Let $\mathfrak{U}$ be an idempotent algebra with no essentially binary polynomial and with essentially ternary and quaternary polynomials. Then the sequence

$$
p_{3}(\mathfrak{u}), p_{4}(\mathfrak{u}), \cdots, p_{n}(\mathfrak{u}), \cdots
$$

is strictly increasing, that is, for all $n \geqq 2$

$$
p_{n}(\mathfrak{u})+1 \leqq p_{n+1}(\mathfrak{U}) \text {. }
$$

The proof starts in $\$ 2$ where a lemma of $\mathrm{K}$. Urbanik is modified to show that the proof splits naturally into three cases. $\$ \S 3$ and 4 handle the first two cases. In $\$ 5$ the third case is analyzed and it is proved that it splits into two further cases that are settled in $\$ \S 6$ and 7. In each of these sections examples are provided that the case under consideration is not void.

For the undefined concepts and basic results the reader is referred to $[2]$.

Examples of algebras satisfying the conditions of the Theorem abound. On a two element Boolean algebra $\{0,1\}$ the operation $(x \wedge y) \vee(y \wedge z) \vee(z \wedge x)$ defines such an algebra.

2. The classification. An algebra $\mathfrak{U}=\langle A ; F\rangle$ is idempotent if every operation $f \in F$ has type (arity) $>0$, and $f(a, \cdots, a)=a$ for all $a \in A$. All algebras considered in this paper are assumed to have more than one element. An $n$-ary polynomial $p$ of $\mathfrak{u}$ (that is, an $n$-ary function or $A$ composed from functions in $F$ ) depends on $x_{i}$ $(1 \leqq i \leqq n)$ if there exist $a_{1}, \cdots, a_{n}, a_{i}^{\prime} \in A$ with $p\left(a_{1}, \cdots, a_{i}, \cdots, a_{n}\right) \neq$ $p\left(a_{1}, \cdots, a_{i}^{\prime}, \cdots, a_{n}\right) ; p$ is essentially $n$-ary, if $p$ depends on $x_{1}, \cdots, x_{n}$. For $n \geqq 2$, let $p_{n}(\mathfrak{l l})$ denote the number of essentially $n$-ary polynomials.

In this paper we shall deal exclusively with idempotent algebras satisfying

$$
p_{2}(\mathfrak{U})=0, p_{3}(\mathfrak{U}) \neq 0, \text { and } \quad p_{4}(\mathfrak{U}) \neq 0 \text {. }
$$

The sequence $\left\langle p_{n}(\mathfrak{U})\right\rangle$ is strictly increasing because $\mathfrak{U}$ must have 
essentially ternary polynomials with very nice properties. This will be used to classify all algebras satisfying these conditions.

A ternary (idempotent) polynomial $p$ is called a minority polynomial if

$$
p(x, x, y)=p(x, y, x)=p(y, x, x)=y ;
$$

$p$ is a majority polynomial, if

$$
p(x, x, y)=p(x, y, x)=p(y, x, x)=x ;
$$

$p$ is a first projection polynomial, if

$$
p(x, x, y)=p(x, y, x)=p(x, y, y)=x .
$$

Observe that a minority or majority ternary polynomial is essentially ternary.

Lemma 1. Let $p(x, y, z)$ be an essentially ternary polynomial satisfying $p(x, y, y)=y$. Then one of $p(z, y, x)$ and $p(y, x, z)$ is an essentially ternary first projection polynomial or one of $p(x, y, z)$ and $p(p(x, y, z), y, z)$ is a majority polynomial.

This statement can be verified by easy computation, observing that $p(y, x, y)=x$ or $y, p(y, y, x)=x$ or $y$, and considering the four cases separately. This argument is the first half of the proof of Lemma 3 of K. Urbanik [6].

THEOREM 2. Let $\mathfrak{u}$ be an idempotent algebra satisfying $p_{2}(\mathfrak{U})=0$ and $p_{3}(\mathfrak{U}) \neq 0$. Then $\mathfrak{U}$ satisfies one (or more) of the following three conditions:

(a) $\mathfrak{u}$ has a ternary majority polynomial;

(b) $\mathfrak{u}$ has an essentially ternary first projection polynomial;

(c ) all essentially ternary polynomials of $\mathfrak{u}$ are minority polynomials.

Proof. Since $p_{3}(\mathfrak{u}) \neq 0, \mathfrak{U}$ has an essentially ternary polynomial $p$. Since $\mathfrak{U}$ is idempotent and $p_{2}(\mathfrak{U})=0, p(x, y, y)=x$ or $y, p(y, x, y)=x$ or $y$, and $p(y, y, x)=x$ or $y$. If the second alternative occurs for any essentially ternary $p$, say $p(x, y, y)=y$, then by Lemma $1, p(z, y, x)$ or $p(y, x, z)$ is an essentially ternary first projection polynomial, or one of $p(x, y, z)$ and $p(p(x, y, z), y, z)$ is a majority polynomial. Thus $\mathfrak{U}$ satisfies (a) or (b). This conclusion cannot be drawn only if for any essentially ternary polynomial $p$ we have $p(x, y, y)=p(y, x, y)=$ $p(y, y, x)=x$, which is (c). 
3. Majority polynomial. Algebras satisfying condition (a) of Theorem 2 shall be handled in this section.

Theorem 3. Let $\mathfrak{U}$ be an idempotent algebra satisfying $p_{2}(\mathfrak{U})=0$. If $\mathfrak{U}$ has a ternary majority polynomial $f$, then

$$
p_{n}(\mathfrak{U})+1 \leqq p_{n+1}(\mathfrak{I})
$$

for $n \geqq 2$.

Proof. For any $n$-ary polynomial $p$ define an $(n+1)$-ary polynomial $p F$ :

$$
p F=f\left(p\left(x_{1}, \cdots, x_{n}\right), p\left(x_{1}, \cdots, x_{n-1}, x_{n+1}\right), p\left(x_{1}, \cdots, x_{n-1}, x_{1}\right)\right) .
$$

Let $f_{3}=f$ and for $n \geqq 3$ define recursively:

$$
f_{n+1}=f_{n} F \text {. }
$$

Finally, we define an $(n+1)$-ary polynomial $g$ :

$$
g=f\left(f_{n}\left(x_{1}, \cdots, x_{n}\right), f_{n}\left(x_{1}, \cdots, x_{n-1}, x_{n+1}\right), x_{2}\right) .
$$

Now we make the following claims:

(i ) For $n \geqq 3$,

$$
f_{n}\left(x_{1}, \cdots, x_{n-1}, x_{1}\right)=x_{1} .
$$

(ii) For $n \geqq 3$,

$$
f_{n}\left(x_{1}, x_{2}, \cdots, x_{2}\right)=x_{2} .
$$

(iii) If the polynomial $p$ is essentially $n$-ary, then $p F$ is essentially $(n+1)$-ary.

(iv) $f_{n}$ is essentially $n$-ary.

(v) $p F=q F$ implies $p=q$.

(vi) $g$ is essentially $(n+1)$-ary.

(vii) $g=p F$ for no polynomial $p$.

Statements (i)-(vii) easily imply the statement of Theorem 3. Indeed, consider the set

$\{g\} \cup\{p F \mid p$ is an essentially $n$-ary polynomial of $\mathfrak{u}\}$.

By (iii) and (vi) all elements of this set are essentially $(n+1)$-ary polynomials. (vii) shows that the union is a disjoint union, and so by $(\mathrm{v})$ the set has $p_{n}(\mathfrak{U})+1$ elements. Thus, $p_{n+1}(\mathfrak{U}) \geqq p_{n}(\mathfrak{U})+1$.

Proof of (i). For $n=3 f_{3}=f$ is a majority polynomial, hence $f_{3}\left(x_{1}, x_{2}, x_{1}\right)=x_{1}$. Proceeding by induction, if $f_{n}\left(x_{1}, \cdots, x_{n-1}, x_{1}\right)=x_{1}$, then 


$$
\begin{aligned}
& f_{n+1}\left(x_{1}, \cdots, x_{n}, x_{1}\right) \\
& \quad=f\left(f_{n}\left(x_{1}, \cdots, x_{n}\right), f_{n}\left(x_{1}, \cdots, x_{n-1}, x_{1}\right), f_{n}\left(x_{1}, \cdots, x_{n-1}, x_{1}\right)\right) \\
& \quad=f\left(f_{n}\left(x_{1}, \cdots, x_{n}\right), x_{1}, x_{1}\right)=x_{1} .
\end{aligned}
$$

Proof of (ii). For $n=3$ (ii) is trivial. By induction, if $f$

$$
f_{n}\left(x_{1}, x_{2}, \cdots, x_{2}\right)=x_{2},
$$

then

$$
\begin{aligned}
& f_{n+1}\left(x_{1}, x_{2}, \cdots, x_{2}\right) \\
& \quad=f\left(f_{n}\left(x_{1}, x_{2}, \cdots, x_{2}\right), f_{n}\left(x_{1}, x_{2}, \cdots, x_{2}\right), f_{n}\left(x_{1}, x_{2}, \cdots, x_{2}, x_{1}\right)\right) \\
& \quad=f\left(x_{2}, x_{2}, x_{1}\right)=x_{2}
\end{aligned}
$$

Proof of (iii). Setting $x_{n}=x_{n+1}$ in $p F$ we get $p$, since $f$ is a majority polynomial. Hence $p F$ depends on $x_{1}, \cdots, x_{n-1}$ and on one or both of $x_{n}$ and $x_{n+1}$. Since $p F$ is symmetric in $x_{n}$ and $x_{n+1}$ in any two element subalgebra the first possibility cannot occur, hence $p F$ is essentially $(n+1)$-ary.

Proof of (iv). Trivial induction using (iii).

Proof of $(\mathrm{v}) . \quad p F$ with $x_{n}=x_{n+1}$ yields $p$, from which the statement follows.

Proof of (vi). Same as the proof of (iv).

Proof of (vii). Let $g=p F$. Setting $x_{n}=x_{n+1}$ we conclude that $f_{n}=p$. Thus $g=f_{n} F=f_{n+1}$, in other words,

$$
\begin{aligned}
& f\left(f_{n}\left(x_{1}, \cdots, x_{n}\right), f_{n}\left(x_{1}, \cdots, x_{n-1}, x_{n+1}\right), f_{n}\left(x_{1}, \cdots, x_{n-1}, x_{1}\right)\right) \\
& \quad=f\left(f_{n}\left(x_{1}, \cdots, x_{n}\right), f_{n}\left(x_{1}, \cdots, x_{n-1}, x_{n+1}\right), x_{2}\right) .
\end{aligned}
$$

Setting $x_{1}=x_{n+1}$ and using (i) and that $f$ is majority we get

$$
x_{1}=f\left(f_{n}\left(x_{1}, \cdots, x_{n}\right), x_{1}, x_{2}\right) .
$$

Finally, setting $x_{2}=x_{3}=\cdots=x_{n}$ and using (ii) we obtain $x_{1}=x_{2}$, a contradiction, proving (vii).

An example of an algebra satisfying the conditions of Theorem 3 was given in $\S 1$. Further examples are easy to construct.

4. First projections polynomial. In this section the Theorem is proved, in a somewhat sharper form, for algebras having an essentially ternary first projection polynomial. 
Theorem 4. Let $\mathfrak{u}$ be an idempotent algebra with $p_{2}(\mathfrak{u})=0$. If $\mathfrak{U}$ has an essentially ternary first projection polynomial $f$, then for $n \geqq 3$

$$
(n-1) p_{n}(\mathfrak{u}) \leqq p_{n+1}(\mathfrak{u})
$$

REMARK. Since, for $n \geqq 3,(n-1) p_{n}(\mathfrak{u}) \geqq 2 p_{n}(\mathfrak{u}) \geqq p_{n}(\mathfrak{U})+1$, Theorem 4 is stronger than the corresponding special case of the Theorem.

Proof. For an $n$-ary polynomial $p$ and $1 \leqq i \leqq n$ set

$$
p F_{i}=f\left(p\left(x_{1}, \cdots, x_{n}\right), x_{i}, x_{n+1}\right) .
$$

Then we make the following claims:

(i) $p F_{i}=q F_{i}$ implies $p=q$.

(ii) If $i \neq j$, then $p F_{i} \neq q F_{j}$.

(iii) $p F_{i}$ depends on $x_{1}, \cdots, x_{n}$.

Since substituting $x_{i}=x_{n+1}$ in $p F_{i}$ yields $p$, we see that if $p F_{i}$ is not essentially $(n+1)$-ary, then by (iii) $p F_{i}=p$. By (ii), $p F_{i} \neq$ $p F_{j}$ if $i \neq j$; hence for $i \neq j$ we cannot have both $p F_{i}$ and $p F_{j}$ not essentially $(n+1)$-ary. Thus for an essentially $n$-ary $p$

$$
\left\{p F_{i} \mid i=1,2, \cdots, n\right\}
$$

contains at least $n-1$ essentially $(n+1)$-ary polynomials. Furthermore, by (i) and (ii) the sets

$$
\left\{p F_{i} \mid i=1,2, \cdots, n\right\} \text { and }\left\{q F_{i} \mid i=1,2, \cdots, n\right\}
$$

are disjoint if $p$ and $q$ are distinct essentially $n$-ary polynomials, from which Theorem 4 follows trivially.

Proof of (i). $\quad p F_{i}$ with $x_{i}=x_{n+1}$ yields $p$, hence (i) is trivial.

Proof of (ii). Let us assume that $i \neq j$ and $p F_{i}=q F_{j}$, that is,

$$
f\left(p\left(x_{1}, \cdots, x_{n}\right), x_{i}, x_{n+1}\right)=f\left(q\left(x_{1}, \cdots, x_{n}\right), x_{j}, x_{n+1}\right) .
$$

Set $x=x_{k}$ for $k \neq i, 1 \leqq k \leqq n$, in this identity; since $p_{2}(\mathfrak{u})=0$ after the substitution $p=x$ or $x_{i}$ and $q=x$ or $x_{i}$. The four possibilities yield the following identities:

$$
\begin{aligned}
& f\left(x, x_{j}, x_{n+1}\right)=f\left(x, x, x_{n+1}\right), \\
& f\left(x, x_{j}, x_{n+1}\right)=f\left(x_{j}, x, x_{n+1}\right), \\
& f\left(x, x_{j}, x_{n+1}\right)=f\left(x, x, x_{n+1}\right), \\
& f\left(x, x_{j}, x_{n+1}\right)=f\left(x_{j}, x, x_{n+1}\right) .
\end{aligned}
$$


The first and third contradict that $f$ is essentially ternary, while the second and fourth mean that $f$ is symmetric in its first and second variable, contradicting that $f$ is a first projection polynomial.

Proof of (iii). Setting $x_{i}=x_{n+1}$ in $p F_{i}$ gives $p$, hence $p F_{i}$ depends on $x_{1}, \cdots, x_{i-1}, x_{i+1}, \cdots, x_{n}$. Assume that $p F_{i}$ does not depend on $x_{i}$. Then

$$
\begin{aligned}
& f\left(p\left(x_{1}, \cdots, x_{n}\right), x_{i}, x_{n+1}\right) \\
& \quad=f\left(p\left(x_{1}, \cdots, x_{i-1}, x_{n+1}, x_{i+1}, \cdots, x_{n}\right), x_{n+1}, x_{n+1}\right) \\
& \quad=p\left(x_{1}, \cdots, x_{i-1}, x_{n+1}, x_{i+1}, \cdots, x_{n}\right) .
\end{aligned}
$$

Substituting $x=x_{j}$ for $j \neq i, 1 \leqq j \leqq n$ and using $p_{2}(\mathfrak{u})=0$ we get one of

$$
\begin{aligned}
& f\left(x, x_{i}, x_{n+1}\right)=x, \\
& f\left(x_{i}, x_{i}, x_{n+1}\right)=x_{n+1} .
\end{aligned}
$$

The first contradicts that $f$ is essentially ternary, while the second is $x_{i}=x_{n+1}$, a contradiction.

An example of an algebra satisfying the condition of Theorem 4 can be defined on the two element set $\{0,1\}$ taking

$$
x+(x+y)(x+z)(y+z)
$$

as operation

$$
\left(u+v=\left(u \wedge v^{\prime}\right) \vee\left(u^{\prime} \wedge v\right)\right)
$$

Taking both

$$
(x \wedge y) \vee(y \wedge z) \vee(z \wedge x) \text { and } x+(x+y)(x+z)(y+z)
$$

as operations we get an algebra satisfying the conditions of Theorems 3 and 4.

Note that in Theorems 3 and $4 p_{4}(\mathfrak{U}) \neq 0$ follows from the assumptions.

5. The second classification. In this and the subsequent sections we consider an idempotent algebra $\mathfrak{U}$ with $p_{2}(\mathfrak{l})=0$ in which all essentially ternary polynomials are minority polynomials.

Lemma 5. $\mathfrak{u}$ has exactly one essentially ternary polynomial.

Proof. Let $f$ and $g$ be essentially ternary polynomials, and consider the polynomial $f(g(x, y, z), y, z)=h$. Then $h(x, y, x)=f(g(x, y, x), y, x)=$ $f(y, y, x)=x$. Thus $h$ cannot be essentially ternary, because it is not 
minority. Due to $p_{2}(\mathfrak{u})=0, h(x, y, z)=x$, or $y$, or $z$. $h(x, y, x)=x$ eliminates $h=y$. Furthermore,

$$
h(x, x, z)=f(g(x, x, z), x, z)=f(z, x, z)=x,
$$

eliminating $h=z$. Hence, $h=x$, that is, we proved the identity

$$
f(g(x, y, z), y, z)=x \text {. }
$$

Now let $a, a^{\prime}, b, c \in A$ and $f(a, b, c)=f\left(a^{\prime}, b, c\right)$. Then

$$
a=f(f(a, b, c), b, c)=f\left(f\left(a^{\prime}, b, c\right), b, c\right)=a^{\prime},
$$

by the above identity (used with $f=g$ )

$$
(a=) f(f(a, b, c), b, c)=(a=) f(g(a, b, c), b, c),
$$

and so by the above remark, $f(a, b, c)=g(a, b, c)$, proving that $f=g$, completing the proof of Lemma 5 .

The only essentially ternary polynomial shall be denoted by $f$. Keep in mind that

$$
f(f(x, y, z), y, z)=x,
$$

and that $f$ is fully symmetric.

The next important step is again due to K. Urbanik. We call a ternary function $g$ on $A$ a Boolean group reduct if a Boolean group operation + can be defined on $A$ (i.e., $\langle A ;+\rangle$ is an abelian group satisfying $2 x=0)$ such that $g(x, y, z)=x+y+z$. The proof of the next lemma is identical with the proof of Lemma 5 of K. Urbanik [6].

LEMmA 6. $f$ is a Boolean group reduct if and only if

$$
f(f(x, y, z), x, u)
$$

does not depend on $x$. If this is the case $+i$ s defined by fixing an arbitrary element $0 \in A$ and $x+y=f(x, y, 0)$.

Accordingly, the proof of the Theorem in the minority polynomial case splits into two completely different cases according to whether or not $f(f(x, y, z), x, u)$ depends on $x$.

6. The minority polynomial is not a Boolean group reduct.

THEOREM 7. Let $\mathfrak{u}$ be an idempotent algebra satisfying $p_{2}(\mathfrak{H})=0$. Let $f$ be the unique essentially ternary minority polynomial of $\mathfrak{u}$. If $f(f(x, y, z), x, u)$ depends on $x$, then for $n \geqq 2$

$$
p_{n}(\mathfrak{U})+1 \leqq p_{n+1}(\mathfrak{U}) .
$$


Proof. We define $f_{3}=f$ and, inductively, for $n \geqq 3$

$$
f_{n+1}=f\left(f_{n}\left(x_{1}, \cdots, x_{n}\right), x_{1}, x_{n+1}\right) .
$$

For an $n$-ary polynomial $p$ and $2 \leqq i \leqq n$ we set

$$
\begin{aligned}
& p G_{1}=f\left(p\left(x_{1}, \cdots, x_{n}\right), x_{1}, x_{n+1}\right), \\
& p G_{i}=p\left(x_{1}, x_{2}, \cdots, x_{i-1}, f\left(x_{i}, x_{1}, x_{n+1}\right), x_{i+1}, \cdots, x_{n}\right) .
\end{aligned}
$$

Observe that $p G_{i}$ with $x_{1}=x_{n+1}$ yields $p$.

We make the following claims:

(i) $f_{n}$ is essentially $n$-ary, and

$$
\begin{aligned}
& f_{3}\left(x_{1}, x_{2}, x_{2}\right)=x_{1}, f_{4}\left(x_{1}, x_{2}, x_{2}, x_{4}\right)=x_{4} . \\
& f_{n}\left(x_{1}, x_{2}, x_{2}, x_{4}, \cdots, x_{n}\right)=f_{n-2}\left(x_{1}, x_{4}, \cdots, x_{n}\right) \text { for } n \geqq 5 .
\end{aligned}
$$

(ii) If $p$ and $q$ are essentially $n$-ary polynomials and $1 \leqq i, j \leqq n$, then $p G_{i}=q G_{j}$ implies $p=q$.

(iii) For an essentially $n$-ary polynomial $p$, at least one of $p G_{1}, \cdots, p G_{n}$ is essentially $(n+1)$-ary. (iii),

Using (i)-(iii) it is easy to prove Theorem 7. Indeed, by (ii) and

$$
P=\left\{p G_{i} \mid p \text { is essentially } n \text {-ary, } i=1, \cdots, n\right\}
$$

contains at least $p_{n}(\mathfrak{Z})$ essentially $(n+1)$-ary polynomials. By (i),

$$
g=f_{n+1}\left(x_{2}, x_{1}, x_{n+1}, x_{3}, x_{4}, \cdots, x_{n}\right)
$$

is also essentially $(n+1)$-ary. If $g \in P$, that is,

$$
g=p G_{i},
$$

for some essentially $n$-ary $p$ and $1 \leqq i \leqq n$, then the substitution $x_{1}=x_{n+1}$ yields

$$
f_{n+1}\left(x_{2}, x_{1}, x_{1}, x_{3}, \cdots, x_{n}\right)=p\left(x_{1}, x_{2}, \cdots, x_{n}\right) .
$$

By the second part of (i) the left-hand side does not depend on $x_{1}$ while the right-hand side does, a contradiction. Thus $g \notin P$, and so $P \cup\{g\}$ contains at least $p_{n}(\mathfrak{A})+1$ essentially $(n+1)$-ary polynomials, proving Theorem 7 .

Proof of (i). We start by proving the formulas in (i). Obviously,

$$
f_{3}\left(x_{1}, x_{2}, x_{2}\right)=x_{1}
$$

and

$$
f_{4}\left(x_{1}, x_{2}, x_{2}, x_{4}\right)=f\left(f_{3}\left(x_{1}, x_{2}, x_{2}\right), x_{1}, x_{4}\right)=f\left(x_{1}, x_{1}, x_{4}\right)=x_{1} .
$$


Thus, for $n \geqq 5$, by induction,

$$
\begin{aligned}
f_{n}\left(x_{1}, x_{2}, x_{2}, x_{4}, \cdots, x_{n}\right) & =f\left(f_{n-1}\left(x_{1}, x_{2}, x_{2}, x_{4}, \cdots, x_{n-1}\right), x_{1}, x_{n}\right) \\
& =f\left(f_{n-3}\left(x_{1}, x_{4}, \cdots, x_{n-1}\right), x_{1}, x_{n}\right) \\
& =f_{n-2}\left(x_{1}, x_{4}, \cdots, x_{n}\right) .
\end{aligned}
$$

(For $n=5$ interpret $f_{n-3}$ as $x_{1}$.)

$f_{3}$ is essentially ternary by assumption. $f_{4}\left(x_{1}, x_{2}, x_{3}, x_{1}\right)=f_{3}\left(x_{1}, x_{2}, x_{3}\right)$, hence $f_{4}$ depends on $x_{2}, x_{3}$. By assumption, $f_{4}$ depends on $x_{1}$. Finally, $f_{4}\left(x_{1}, x_{2}, x_{2}, x_{4}\right)=x_{4}$, hence $f_{4}$ depends on $x_{4}$. Thus $f_{4}$ is essentially 4-ary. Proceeding by induction for $n \geqq 5, f_{n}$ with $x_{1}=x_{n}$ yields $f_{n-1}\left(x_{1}, \cdots, x_{n-1}\right)$, hence $f_{n}$ depends on $x_{2}, \cdots, x_{n-1}$. Finally, $f_{n}$ with $x_{2}=x_{3}$ gives

$$
f_{n-2}\left(x_{1}, x_{4}, \cdots, x_{n}\right) \text {, }
$$

which depends on $x_{1}$ and $x_{n}$, hence $f_{n}$ depends on $x_{1}$ and $x_{n}$.

Proof of (ii). Obvious; by setting $x_{1}=x_{n+1}$ in $p G_{i}=q G_{j}$ we get $p=q$.

Proof of (iii). $p G_{i}$ with $x_{1}=x_{n+1}$ gives $p\left(x_{1}, \cdots, x_{n}\right)$, hence $p G_{i}$ depends on $x_{2}, \cdots, x_{n}$. Furthermore, $p G_{1}$ with $x_{1}=x_{2}=\cdots=x_{n}$ gives $f\left(x_{1}, x_{1}, x_{n+1}\right)=x_{n+1}$ and $p G_{i}(i>1)$ with $x_{1}=x_{n+1}$ gives

$$
p\left(x_{1}, \cdots, x_{i-1}, x_{n+1}, x_{i+1}, \cdots, x_{n}\right),
$$

hence all $p G_{i}, 1 \leqq i \leqq n$ depend on $x_{n+1}$. Thus if none of $p G_{1}, \cdots, p G_{n}$ is essentially $(n+1)$-ary then none of them depend on $x_{1}$.

So assume that none of $p G_{1}, \cdots, p G_{n}$ depend on $x_{1}$. Then by substituting $x_{1}=x_{n+1}$ in $p G_{1}$ we get the identity

$$
f\left(p\left(x_{1}, \cdots, x_{n}\right), x_{1}, x_{n+1}\right)=p\left(x_{n+1}, \cdots, x_{n}\right) .
$$

For $i>1$ we obtain

$$
\begin{aligned}
p\left(x_{n+1}, x_{2}, \cdots, x_{i}, \cdots, x_{n}\right) & =p\left(x_{1}, \cdots, x_{i-1}, f\left(x_{i}, x_{1}, x_{n+1}\right), \cdots, x_{n}\right) \\
& =p\left(x_{1}, \cdots, x_{i}, \cdots, x_{n}\right) .
\end{aligned}
$$

Since this holds for all $i>1, p$ is symmetric. Then, using the identity (*) repeatedly we obtain

$$
\begin{aligned}
p\left(x_{1}, \cdots, x_{n}\right) & =p\left(x_{n}, x_{n-1}, \cdots, x_{1}\right)=f\left(p\left(x_{1}, x_{n-1}, \cdots, x_{1}\right), x_{1}, x_{n}\right) \\
& =f\left(f\left(p\left(x_{1}, x_{1}, x_{n-2}, \cdots, x_{1}\right), x_{1}, x_{n-1}\right), x_{1}, x_{n}\right) \\
& =f\left(\cdots f\left(p\left(x_{1}, x_{1}, \cdots, x_{1}\right), x_{1}, x_{2}\right) \cdots\right) \\
& =f_{n}\left(x_{1}, x_{2}, \cdots, x_{n}\right) .
\end{aligned}
$$

Hence, $p=f_{n}$. But then $\left({ }^{*}\right)$ states that $f_{n+1}\left(x_{1}, \cdots, x_{n+1}\right)$ does not depend on $x_{1}$, a contradiction. 
Idempotent algebras satisfying $p_{2}=0$ and having a unique ternary minority polynomial can be constructed from Steiner quadruple systems and vice versa. A Steiner quadruple system is a set $A$ and a set $S$ of four element subsets of $A$ with the property that any three element subset of $A$ belongs to one and only one member of $S$. For such a system define an algebra $\langle A ; f\rangle$ as follows:

$f$ is a minority function and for three distinct elements $a, b, c \in A$ there is a unique member $B \in S$ with $a, b, c \in B$; let $B=\{a, b, c, d\}$; set $f(a, b, c)=d$.

Conversely, if an idempotent algebra $\langle A ; F\rangle$ satisfies $p_{2}=0$ and $f$ is the unique ternary minority polynomial, then set

$$
S=\{\{a, b, c, f(a, b, c)\}|a, b, c \in A,|\{a, b, c\} \mid=3\} .
$$

Then this defines a Steiner quadruple system.

The smallest Steiner quadruple system which is associated with an algebra satisfying the conditions of Theorem 7 can be defined on $A=\{1,2, \cdots, 10\}$ as follows (see [1]):

$\begin{array}{rrrrrrrr}1 & 2 & 3 & 10 & 1 & 3 & 5 & 8 \\ 4 & 5 & 6 & 10 & 4 & 5 & 7 & 8 \\ 7 & 8 & 9 & 10 & 1 & 2 & 6 & 9 \\ 1 & 4 & 7 & 10 & 2 & 3 & 8 & 9 \\ 2 & 5 & 8 & 10 & 1 & 5 & 6 & 7 \\ 3 & 6 & 9 & 10 & 1 & 3 & 7 & 9 \\ 1 & 5 & 9 & 10 & 2 & 4 & 6 & 8 \\ 2 & 6 & 7 & 10 & 1 & 2 & 7 & 8 \\ 3 & 4 & 8 & 10 & 3 & 4 & 5 & 9 \\ 3 & 5 & 7 & 10 & 2 & 3 & 5 & 6 \\ 2 & 4 & 9 & 10 & 1 & 4 & 8 & 9 \\ 1 & 6 & 8 & 10 & 1 & 3 & 4 & 6 \\ 2 & 3 & 4 & 7 & 2 & 5 & 7 & 9 \\ 5 & 6 & 8 & 9 & 1 & 2 & 4 & 5 \\ 4 & 6 & 7 & 9 & 3 & 6 & 7 & 8\end{array}$

Obviously, the associated $f$ is not a Boolean group reduct since $|A|=10$ is not a power of two. However, an example, which is due to N.S. Mendelsohn, shows that even if $|A|$ is a power of two, examples of algebras satisfying the conditions of Theorem 7 can be defined on $A$ provided that $|A| \geqq 16$. Let $A=\{1,2, \cdots, 16\}$ and let $S$ be given by the following table:

$\begin{array}{rrrrrrrr}1 & 2 & 3 & 4 & 2 & 3 & 5 & 8 \\ 1 & 2 & 5 & 6 & 2 & 3 & 9 & 12 \\ 1 & 2 & 7 & 8 & 2 & 3 & 13 & 16 \\ 1 & 2 & 9 & 10 & 2 & 3 & 6 & 7\end{array}$




$\begin{array}{rrrrrrrr}1 & 2 & 11 & 12 & 2 & 3 & 10 & 11 \\ 1 & 2 & 13 & 14 & 2 & 3 & 14 & 15 \\ 1 & 2 & 15 & 16 & 2 & 4 & 5 & 7 \\ 1 & 3 & 5 & 7 & 2 & 4 & 8 & 9 \\ 1 & 3 & 6 & 8 & 2 & 4 & 10 & 12 \\ 1 & 3 & 9 & 11 & 2 & 4 & 13 & 15 \\ 1 & 3 & 10 & 12 & 2 & 4 & 6 & 16 \\ 1 & 3 & 13 & 15 & 2 & 4 & 11 & 14 \\ 1 & 3 & 14 & 16 & 2 & 5 & 9 & 15 \\ 1 & 4 & 5 & 8 & 2 & 5 & 10 & 16 \\ 1 & 4 & 6 & 7 & 2 & 5 & 11 & 13 \\ 1 & 4 & 9 & 12 & 2 & 5 & 12 & 14 \\ 1 & 4 & 10 & 11 & 2 & 6 & 8 & 14 \\ 1 & 4 & 13 & 16 & 2 & 6 & 10 & 15 \\ 1 & 4 & 14 & 15 & 2 & 6 & 9 & 11 \\ 1 & 5 & 9 & 13 & 2 & 6 & 12 & 13 \\ 1 & 5 & 10 & 14 & 2 & 7 & 9 & 13 \\ 1 & 5 & 11 & 15 & 2 & 7 & 10 & 14 \\ 1 & 5 & 12 & 16 & 2 & 7 & 11 & 15 \\ 1 & 6 & 9 & 14 & 2 & 7 & 12 & 16 \\ 1 & 6 & 10 & 13 & 2 & 8 & 10 & 13 \\ 1 & 6 & 11 & 16 & 2 & 8 & 12 & 15 \\ 1 & 6 & 12 & 15 & 2 & 8 & 11 & 16 \\ 1 & 7 & 9 & 15 & 2 & 9 & 14 & 16 \\ 1 & 7 & 10 & 16 & 3 & 4 & 5 & 6 \\ 1 & 7 & 11 & 13 & 3 & 4 & 7 & 8 \\ 1 & 7 & 12 & 14 & 3 & 4 & 9 & 13 \\ 1 & 8 & 9 & 16 & 3 & 4 & 10 & 14 \\ 1 & 8 & 10 & 15 & 3 & 4 & 11 & 12 \\ 1 & 8 & 11 & 14 & 3 & 4 & 15 & 16 \\ 1 & 8 & 12 & 13 & 3 & 5 & 9 & 14 \\ 3 & 5 & 10 & 15 & 5 & 6 & 11 & 14 \\ 3 & 5 & 11 & 16 & 5 & 7 & 9 & 10 \\ 3 & 5 & 12 & 13 & 5 & 7 & 11 & 12 \\ 3 & 6 & 9 & 10 & 5 & 7 & 13 & 14 \\ 3 & 6 & 11 & 15 & 5 & 7 & 15 & 16 \\ 3 & 6 & 12 & 16 & 5 & 8 & 9 & 12 \\ 3 & 6 & 13 & 14 & 5 & 8 & 13 & 16 \\ 3 & 7 & 9 & 16 & 5 & 8 & 10 & 11 \\ 3 & 7 & 10 & 13 & 5 & 8 & 14 & 15 \\ 3 & 7 & 11 & 14 & 6 & 7 & 9 & 12 \\ 3 & 7 & 12 & 15 & 6 & 7 & 13 & 16 \\ 3 & 8 & 9 & 15 & 6 & 7 & 10 & 11 \\ 3 & 8 & 10 & 16 & 6 & 7 & 14 & 15\end{array}$




$\begin{array}{rrrrrrrr}3 & 8 & 11 & 13 & 6 & 8 & 9 & 13 \\ 3 & 8 & 12 & 14 & 6 & 8 & 15 & 16 \\ 4 & 5 & 9 & 11 & 6 & 8 & 11 & 12 \\ 4 & 5 & 12 & 15 & 6 & 10 & 14 & 16 \\ 4 & 5 & 14 & 16 & 7 & 8 & 9 & 11 \\ 4 & 5 & 10 & 18 & 7 & 8 & 10 & 12 \\ 4 & 6 & 8 & 10 & 7 & 8 & 13 & 15 \\ 4 & 6 & 11 & 13 & 7 & 8 & 14 & 16 \\ 4 & 6 & 12 & 14 & 8 & 9 & 10 & 14 \\ 4 & 6 & 9 & 15 & 9 & 10 & 11 & 12 \\ 4 & 7 & 9 & 14 & 9 & 10 & 13 & 15 \\ 4 & 7 & 10 & 15 & 9 & 11 & 13 & 14 \\ 4 & 7 & 11 & 16 & 9 & 11 & 15 & 16 \\ 4 & 7 & 12 & 13 & 9 & 12 & 13 & 16 \\ 4 & 8 & 11 & 15 & 9 & 12 & 14 & 15 \\ 4 & 8 & 12 & 16 & 10 & 11 & 13 & 16 \\ 4 & 8 & 13 & 14 & 10 & 11 & 14 & 15 \\ 4 & 9 & 10 & 16 & 10 & 12 & 13 & 14 \\ 5 & 6 & 7 & 8 & 10 & 12 & 15 & 16 \\ 5 & 6 & 9 & 16 & 11 & 12 & 13 & 15 \\ 5 & 6 & 10 & 12 & 11 & 12 & 14 & 16 \\ 5 & 6 & 13 & 15 & 13 & 14 & 15 & 16\end{array}$

In this example,

$$
f\left(f\left(x_{1}, x_{2}, x_{3}\right), x_{1}, x_{4}\right)=f\left(x_{1}, x_{2}, f\left(x_{1}, x_{3}, x_{4}\right)\right),
$$

and therefore

$$
x+y=f(x, y, 0)
$$

defines a Boolean group operation for any fixed $0 \in A$. However, $f(x, y, z) \neq x+y+z$. To prove this it suffices by Lemma 6 to illustrate that $f(f(x, y, z), x, u)$ depends on $x$. Indeed, $f(f(1,4,5), 1,6)=3$ and $f(f(9,4,5), 9,6)=2$.

It may be of interest to note that recently C. Treash [5] has solved the word problem for algebras $\langle A ; f\rangle$ of type $\langle 3\rangle$, where $f$ is a minority function and

$$
f(f(x, y, z), y, z)=x .
$$

7. Boolean reducts. In this section we settle the final case of the Theorem.

THEOREM 8. Let $\mathfrak{u}$ be an idempotent algebra satisfying $p_{2}(\mathfrak{U})=0$, $p_{3}(\mathfrak{U}) \neq 0$, and $p_{4}(\mathfrak{H}) \neq 0$, having a unique essentially ternary minority 
polynomial $f$. If $f(f(x, y, z), x, u)$ does not depend on $x$, then

$$
p_{n}(\mathfrak{u})+1 \leqq p_{n+1}(\mathfrak{u})
$$

for $n=2,3, \cdots$.

Proof. By Lemma 6, a Boolean group operation + can be defined on $A$ such that $f(x, y, z)=x+y+z$. Let $p$ be an essentially 4-ary polynomial of $\mathfrak{U}$ (recall that $p_{4}(\mathfrak{U}) \neq 0$ ). It follows from Lemma 6 of [6], that there exists a ternary polynomial $p_{0}$ of $\langle A ; f\rangle$ such that $p(x, y, z, u)=p_{0}(x, y, z, u)$ whenever $x, y, z$, and $u$ are not all distinct. If $p_{0}=x$, then we can conclude that $p$ is an essentially 4-ary first projection polynomial, that is, it satisfies

$p(x, y, z, u)=x$ whenever $x, y, z$, and $u$ are not all distinct. If $p_{0}=y, p_{0}=z$, or $p_{0}=u$, we get a first projection polynomial by permuting the variables of $p$. If $p_{0}=x+y+z$, then $p+y+z$ is the first projection polynomial. Observe that $p+y+z$ is essentially 4-ary, since otherwise $p+y+z$ would be a polynomial of $f$, implying that $p=(p+y+z)+y+z$ is a polynomial of $f$. If $p_{0}=x+y+$ $u, \cdots$ we proceed similarly.

Thus there exists in $\mathfrak{U}$ an essentially 4-ary first projection polynomial $g$. (This statement is a small part of Lemma 7 in [6].)

Now we start our constructions.

Let $p=p\left(x_{1}, \cdots, x_{n}\right)$ be an essentially $n$-ary polynomial, $n \geqq 4$. We construct an $(n+1)$-ary polynomial $\bar{p}$ as follows:

$p(x, x, \cdots, x, y, z)$ is a ternary polynomial of $\mathfrak{u}$, hence it is $x, y, z$, or $x+y+z$;

(1) if $p(x, \cdots, x, y, z)=x$, then $\bar{p}=g\left(p\left(x_{1}, \cdots, x_{n}\right), x_{n-1}, x_{n}, x_{n+1}\right)$;

(2) if $p(x, \cdots, x, y, z)=y$, then $\bar{p}=g\left(p\left(x_{1}, \cdots, x_{n}\right), x_{1}, x_{n}, x_{n+1}\right)$;

(3) if $p(x, \cdots, x, y, z)=z$, then $\bar{p}=g\left(p\left(x_{1}, \cdots, x_{n}\right), x_{1}, x_{n-1}, x_{n+1}\right)$;

(4) if $p(x, \cdots, x, y, z)=x+y+z$, then

$$
\bar{p}=g\left(p\left(x_{1}, \cdots, x_{n}\right)+x_{n-1}+x_{n}, x_{n-1}, x_{n}, x_{n+1}\right)+x_{n-1}+x_{n} .
$$

Furthermore, for $n \geqq 4$ we define $g_{n}$ by recursion: $g_{4}=g$ and

$$
g_{n+1}=g\left(g_{n}, x_{2}, x_{3}, x_{n+1}\right) \text {. }
$$

Then we claim the following:

(i) For an essentially $n$-ary $p$, the polynomial $\bar{p}$ is essentially $(n+1)$-ary.

(ii) If $p$ and $q$ are essentially $n$-ary polynomials and $p \neq q$, then $\bar{p} \neq \bar{q}$.

(iii) For $n \geqq 4, g_{n}\left(x_{1}, \cdots, x_{1}, x_{n-1}, x_{n}\right)=g_{n}\left(x_{1}, x_{2}, x_{3}, \cdots, x_{3}\right)=x_{1}$.

(iv) $g_{n}$ is essentially $n$-ary.

(v) $g_{n+1}=\bar{p}$ for no essentially $n$-ary polynomial $p$.

Now Theorem 8 is clear: 


\section{$\{\bar{p} \mid p$ essentially $n$-ary $\} \cup\left\{g_{n+1}\right\}$}

is a set of $p_{n}(\mathfrak{u})+1$ essentially $(n+1)$-ary polynomials by (i), (ii), (iv), and $(\mathrm{v})$.

In the subsequent proofs Case $1, \cdots$, Case 4 refer to the cases in the definition of $\bar{p}$.

Proof of (i). Case 1. $\bar{p}$ with $x_{n+1}=x_{n}$ yields $p\left(x_{1}, \cdots, x_{n}\right)$, hence $\bar{p}$ depends on $x_{1}, \cdots, x_{n-1}$. The substitution $x_{n+1}=x_{n-1}$ gives that $\bar{p}$ depends on $x_{n}$. Setting $x_{1}=x_{2}=\cdots=x_{n-1}$ in $\bar{p}$ (observe that $p\left(x_{1}, \cdots, x_{1}, x_{n-1}, x_{n}\right)=x_{1}$ by assumption) yields $g\left(x_{1}, x_{n-1}, x_{n}, x_{n+1}\right)$, hence $\bar{p}$ depends on $x_{n+1}$.

Case 2. Use the substitutions

$$
x_{n+1}=x_{1}, x_{n+1}=x_{n} \text {, and } x_{1}=\cdots=x_{n-2} \text {. }
$$

Case 3. Use the substitutions

$$
x_{n+1}=x_{1}, x_{n+1}=x_{n-1} \text {, and } x_{1}=\cdots=x_{n-2} \text {. }
$$

Case 4. Just as in the previous cases,

$$
x_{n+1}=x_{n} \text { and } x_{n+1}=x_{n-1}
$$

establish that $\bar{p}$ depends on $x_{1}, \cdots, x_{n}$. Setting $x_{1}=\cdots=x_{n-2}$ in $\bar{p}$ we get $h=g\left(x_{1}, x_{n-1}, x_{n}, x_{n+1}\right)+x_{n-1}+x_{n}$. Observe that $h+x_{n-1}+x_{n}=$ $g\left(x_{1}, x_{n-1}, x_{n}, x_{n+1}\right)$ depends on $x_{n+1}$, therefore so does $h$. Thus $\bar{p}$ depends on $x_{n+1}$.

Proof of (ii). Set $A_{1}=\{n-1, n, n+1\}, A_{2}=\{1, n, n+1\}, A_{3}=$ $\{1, n-1, n+1\}$, and $A_{4}=\{n-1, n, n+1\}$. If $p$ belongs to Case $i$ and $k, l \in A_{i}, k \neq l$, then $x_{k}=x_{l}$ substituted into $\bar{p}$ yields $p$. Observe that $\left|A_{i} \cap A_{j}\right| \geqq 2$ for $1 \leqq i, j \leqq 4$. Now if $\bar{p}=\bar{q}, p$ belongs to Case $i, q$ to Case $j$, then we can choose $k, l \in A_{i} \cap A_{j}, k \neq l$. Substituting $x_{k}=x_{l}$ into $\bar{p}=\bar{q}$ gives $p=q$.

Proof of (iii). For $n=4, g_{4}\left(x_{1}, x_{1}, x_{3}, x_{4}\right)=g_{n}\left(x_{1}, x_{2}, x_{3}, x_{3}\right)=x_{1}$, since $g_{4}=g$ is a first projection polynomial. Assuming the identities for $n$, we compute:

$$
g_{n+1}\left(x_{1}, \cdots, x_{1}, x_{n}, x_{n+1}\right)=g\left(g_{n}\left(x_{1}, \cdots, x_{1}, x_{n}\right), x_{1}, x_{1}, x_{n+1}\right)=x_{1}
$$

and

$$
g_{n+1}\left(x_{1}, x_{2}, x_{3}, \cdots, x_{3}\right)=g\left(g_{n}\left(x_{1}, x_{2}, x_{3}, \cdots, x_{3}\right), x_{2}, x_{3}, x_{3}\right)=x_{1} .
$$

Proof of (iv). $g_{4}=g$ so the statement is true for $n=4$. Assume it for $n$. Substituting $x_{n+1}=x_{2}$ or $x_{n+1}=x_{3}$ into $g_{n+1}$ yields $g_{n}$, hence 
$g_{n+1}$ depends on $x_{1}, \cdots, x_{n}$. Substituting $x_{3}=x_{4}=\cdots=x_{n}$ into $g_{n+1}$ gives by (iii) $g\left(g_{n}\left(x_{1}, x_{2}, x_{3}, \cdots, x_{3}\right), x_{2}, x_{3}, x_{n+1}\right)=g\left(x_{1}, x_{2}, x_{3}, x_{n+1}\right)$, hence $g_{n+1}$ depends on $x_{n+1}$.

Proof of (v). Observe that $g_{n+1}\left(x_{1}, x_{2}, x_{2}, x_{4}, \cdots, x_{n+1}\right)=x_{1}$. Hence, if $g_{n+1}=\bar{p}$, then $\bar{p}\left(x_{1}, x_{2}, x_{2}, x_{4}, \cdots, x_{n+1}\right)=x_{1}$. Further substituting $x_{n+1}=x_{n}$ or (Case 3) $x_{n+1}=x_{n-1}$, we conclude that

$$
p\left(x_{1}, x_{2}, x_{2}, x_{4}, \cdots, x_{n}\right)=x_{1} .
$$

This is impossible if $p$ belongs to Cases 2 or 3 , and it immediately yields a contradiction in Case 1 (namely, $x_{1}=g\left(x_{1}, x_{n-1}, x_{n}, x_{n+1}\right)$ ) and in Case 4.

\section{REFERENCES}

1. R. D. Carmichael, Groups of Finite Order, Dover Publ. Inc. 1956.

2. G. Grätzer, Universal Algebra, The University Series in Higher Mathematics. D. Van Nostrand Co., Princeton, N. J., 1968.

3. Composition of Functions, Proceedings of the Conference on Universal Algebra, October 1969. Queen's Papers in Pure and Applied Mathematics, No. 25, Queen's University, Kingston, Ont., 1970.

4. G. Grätzer and J. Plonka, On the number of polynomials of an idempotent algebra. I, Pacific J. Math., 32 (1970), 697-709.

5. C. Treash, The Completion of Finite Incomplete Steiner Triple Systems with Applications to Loop Theory.

6. K. Urbanik, On algebraic operations in idempotent algebras, Colloq. Math., 13 (1965), 129-157.

Received May 6, 1972. The work of both authors was supported by the National Research Council of Canada.

The UNIVERSITy of MANitoba,

AND

The Mathematical Research Institute of the Polish Academy of Sciences, Wroclaw, POLAND 



\section{PACIFIC JOURNAL OF MATHEMATICS}

\section{EDITORS}

D. Gilbarg and J. Milgram

Stanford University

Stanford, California 94305

R. A. Beaumont

University of Washington

Seattle, Washington 98105
J. DUGUNDJI* Department of Mathematics

University of Southern California Los Angeles, California 90007

RICHARD ARENS

University of California

Los Angeles, California 90024

\section{ASSOCIATE EDITORS}

E. F. BeCKenbach

B. H. NeUMaNN

F. WOLF

K. Yoshida

\section{SUPPORTING INSTITUTIONS}

UNIVERSITY OF BRITISH COLUMBIA

UNIVERSITY OF SOUTHERN CALIFORNIA

CALIFORNIA INSTITUTE OF TECHNOLOGY

UNIVERSITY OF CALIFORNIA

MONTANA STATE UNIVERSITY

STANFORD UNIVERSITY

UNIVERSITY OF TOKYO

UNIVERSITY OF NEVADA

UNIVERSITY OF UTAH

NEW MEXICO STATE UNIVERSITY

WASHINGTON STATE UNIVERSITY

OREGON STATE UNIVERSITY

UNIVERSITY OF OREGON

OSAKA UNIVERSITY

UNIVERSITY OF WASHINGTON

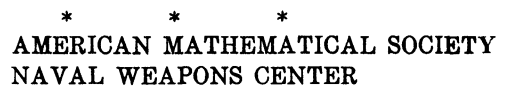

* C. DePrima will replace J. Dugundji until August 1974. 


\section{Pacific Journal of Mathematics}

\section{Vol. 47, No. $1 \quad$ January, 1973}

K. Adachi, Masuo Suzuki and M. Yoshida, Continuation of holomorphic

mappings, with values in a complex Lie group ....................

Michael Aschbacher, A characterization of the unitary and symplectic groups

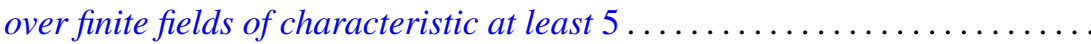

Larry Eugene Bobisud and James Calvert, Energy bounds and virial theorems for abstract wave equations....................................

Christer Borell, A note on an inequality for rearrangements ................

Peter Southcott Bullen and S. N. Mukhopadhyay, Peano derivatives and general

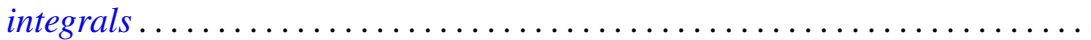

Wendell Dan Curtis, Yu-Lee Lee and Forrest Miller, A class of infinite dimensional subgroups of $\operatorname{Diff}^{r}(X)$ which are Banach Lie groups .........

Paul C. Eklof, The structure of ultraproducts of abelian groups ...............

William Alan Feldman, Axioms of countability and the algebra $C(X) \ldots \ldots \ldots$

Jack Tilden Goodykoontz, Jr., Aposyndetic properties of hyperspaces...........

George Grätzer and J. Płonka, On the number of polynomials of an idempotent algebra. II ...........................................

Alan Trinler Huckleberry, The weak envelope of holomorphy for algebras of

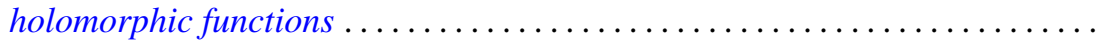

John Joseph Hutchinson and Julius Martin Zelmanowitz, Subdirect sum decompositions of endomorphism rings . . . . . . . . . . . . . . . .

Gary Douglas Jones, An asymptotic property of solutions of

$y^{\prime \prime \prime}+p y^{\prime}+q y=0$.

Howard E. Lacey, On the classification of Lindenstrauss spaces .

Charles Dwight Lahr, Approximate identities for convolution measure algebras.

George William Luna, Subdifferentials of convex functions on Banach

spaces.

Nelson Groh Markley, Locally circular minimal sets. .

Robert Wilmer Miller, Endomorphism rings of finitely generated projective modules

Donald Steven Passman, On the semisimplicity of group rings of linear

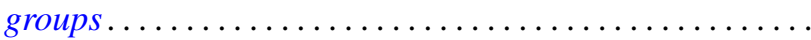

Bennie Jake Pearson, Dendritic compactifications of certain dendritic spaces.

Ryōtarō Satō, Abel-ergodic theorems for subsequences ...... .

Henry S. Sharp, Jr., Locally complete graphs. . .

Harris Samuel Shultz, A very weak topology for the Mikusinski field of

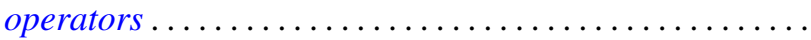

Elena Stroescu, Isometric dilations of contractions on Banach spaces ...

Charles W. Trigg, Versum sequences in the binary system ... . .

William L. Voxman, On the countable union of cellular decompositions of n-manifolds 